\title{
Étude Évaluative De L'application Des Textes Juridiques En Vigueur En Matière De Lutte Contre La Contrefaçon En Côte d'Ivoire
}

\author{
Dr Kazon Diescieu Aubin Sylvère \\ Maitre-Assistant en Criminologie, Université Félix Houphouet Boigny \\ Cocody, UFR Criminologie, Côte d'Ivoire \\ Dr Dagbé Ahodan Stéphane \\ Maitre-Assistant en Criminologie, Université Félix Houphouet Boigny \\ Cocody, UFR Criminologie, Côte d'Ivoire \\ Blé Edwige \\ Doctorante en Criminologie, Université Félix Houphouet-Boigny, UFR \\ Criminologie, Côte d'Ivoire
}

Doi:10.19044/esj.2018.v14n17p195 URL:http://dx.doi.org/10.19044/esj.2018.v14n17p195

\begin{abstract}
This study aim at assessing the application of laws within the fight against counterfeiting in Ivory Coast. The work took place in Abidjan (the economic capital city), precisely in the communes of Plateau and Koumassi and has focused on a sample of 32 respondents selected according to the criteria of intentional sampling method. The data have been collected by direct observation and with the help of a questionnaire; all from the documentary study. As far as the methods of analysis of the data are concerned, the emphasis has been placed on the qualitative analysis. Thus, the results obtained show many difficulties in the application of the legal texts in the field of the fight against counterfeiting. These difficulties result in large part from the legal problems and the enforcement of decisions, the lapsing of certain laws which are more in line with the requirements of international trade and of the inadequacy of some laws. From these results, we propose that the sentences are more dissuasive and the fines very high. The means must be put at the disposal of sworn officers to rigorously enforce the laws. They will need to have coercive powers, or even law enforcement agencies to track down counterfeiters and spurious goods. There must be an honest collaboration between economic operators and agents in charge of making the laws respected.
\end{abstract}

Keywords: Evaluative study, legal texts, counterfeiting, intellectual property 


\section{Résumé}

Cette étude a pour objectif d'évaluer l'application des textes de lois en vigueur en matière de lutte contre la contrefaçon en Côte d'Ivoire. Le travail s'est déroulé à Abidjan (capitale économique), précisément dans les communes du Plateau et de Koumassi et a porté sur un échantillon de 32 enquêtés sélectionnés selon les critères de la méthode d'échantillonnage intentionnel. Les données ont été recueillies par l'observation directe et à l'aide d'un questionnaire; le tout à partir de l'étude documentaire. Pour ce qui est des méthodes d'analyse des données, l'accent a été mis sur l'analyse qualitative. Ainsi, les résultats obtenus montrent bien des difficultés dans l'application des textes juridiques en matière de lutte contre la contrefaçon. Ces difficultés découlent en grande partie des problèmes juridiques et de l'exécution des décisions, de la caducité de certaines lois qui ne sont plus conformes aux exigences du commerce international et de l'insuffisance de certains textes de lois. À partir de ces résultats, nous proposons que les peines soient plus dissuasives et les amendes très élevées. Les moyens doivent être mis à la disposition des agents assermentés pour appliquer rigoureusement les lois. Ceux-ci devront disposer de pouvoirs coercitifs, voire répressifs pour traquer contrefacteurs et marchandises contrefaites. Il faut qu'il y ait une franche collaboration entre les opérateurs économiques et les agents en charge d'appliquer les lois.

Mots clés : Etude évaluative, textes juridiques, contrefaçon, propriété intellectuelle

\section{Introduction}

Lutter contre la contrefaçon est plus qu'une préoccupation majeure dans tous les pays du monde, en particulier dans les États en quête de développement. Ce trafic illicite a pris une proportion telle qu'aucun secteur d'activités économiques n'est épargné (Blé, 2013). Ainsi pour tenter de comprendre la contrefaçon, certains chercheurs se sont intéressés à sa définition. Maitre et Perrino (2007) appréhendent la contrefaçon comme une identité absolue de signes, une similarité entre produits et services et un risque de confusion de la part du consommateur. Pour ces auteurs, le contrefacteur cherche à s'approprier la notoriété d'autrui et à profiter indûment des investissements réalisés par le véritable titulaire des droits de propriété intellectuelle. Harms (2007) va dans le même sens en présentant la contrefaçon comme une forme de tromperie. Un produit de contrefaçon, souligne-t-il, est une chose qui a été fabriquée, copiée ou illégalement imitée aux fins de soutirer de l'argent à des clients crédules ou consentants au préjudice de celui qui avait, seul, le droit de la fabriquer. 
D'autres écrits se sont également intéressés à la diversité des formes de contrefaçon. Dans cette perspective, la contrefaçon touche tous les secteurs d'activité allant des produits agroalimentaires au bâtiment, en passant par les produits pharmaceutiques, l'automobile, le textile, les composants informatiques, les livres, les parfums, les produits cosmétiques, les jouets, les montres, les marques de produits de luxe, les vins et spiritueux, etc (OCDE, 2008). C'est ce que caricature Viala (2008) en indiquant le "boom" de l'industrie de la contrefaçon qui " clone " désormais les produits de grandes consommations, du lait de nourrissons aux pièces détachées en passant par les médicaments.

Avec cette diversité, la contrefaçon est devenue, au fil des années, une formidable lessiveuse à blanchir de l'argent. Elle fait désormais partie intégrante des nouvelles activités des organisations criminelles dont certaines seraient liées aux terrorismes (Delval, 2008). Hyeans (2011) soutient que la contrefaçon constitue une des préoccupations majeures de la sécurité. Cet auteur inscrit la contrefaçon au nombre des facteurs qui affectent la stabilité même du système socio-économique et de ses fondations culturelles, éthiques et morales. Aussi, la contrefaçon profite aux réseaux criminels en contournant les législations ou en s'engouffrant dans ses silences. C'est à bon droit donc que les États choisissent de lutter contre ce phénomène en le criminalisant, car aucun d'entre eux n'en est vraiment épargné. Selon Hyeans, les enjeux pour les produits européens sont éminemment importants, tant en termes de compétitivité de l'économie, que de la protection des consommateurs, de l'environnement et de la santé.

Bhumindr (2012) s'intéresse au mode opératoire des auteurs des contrefaçons. Pour lui, les contrefacteurs profitent de la mondialisation et de l'ampleur des réseaux commerciaux pour avancer " masqués » et brouiller les pistes. Comme le souligne l'Union des fabricants, la mobilité et l'opacité des circuits sont telles que chaque contrefaçon dispose de son propre mode de cheminement et de distribution.

Aujourd'hui, une analyse précise de ce phénomène, de son origine et de sa diversité met en évidence ses répercussions négatives sur l'économie et la société. Les conséquences sont souvent très lourdes.

D'abord pour les entreprises, elle engendre la perte de parts conséquentes de marché, la destruction des emplois, et le ternissement de l'image de marque. "Pendant longtemps, on a eu tendance à sous-estimer les véritables dangers liés à la contrefaçon: dangers économiques, car la contrefaçon détruit les emplois; mas aussi dangers pour les consommateurs qui sont trompés sur la véritable nature des produits " (Lagarde, 2009). Soutenant cette même thèse, en ce qui concerne le marché ivoirien, l'industriel Conquet (2013) révèle, dans le cadre du secteur du sucre, que 40.000 tonnes en provenance de l'extérieur entrent frauduleusement en Côte 
d'Ivoire chaque année. Ces marchandises, transportées dans des emballages brésiliens ou indiens, auraient occasionné, en cinq ans, un recul de $30 \%$ de parts de marché pour le sucre ivoirien, soit une perte annuelle de 20 milliards de francs $\mathrm{Cfa}$ pour la filière. Ces pertes constituent une manne très importante pour l'État en termes d'évasion fiscale dont le coût économique et social, évalué par l'Ong 'Stop à la fraude fiscale et à la contrefaçon sans frontière", est alarmant. "La Côte d'Ivoire perd 500 milliards de francs CFA à cause de la contrefaçon ", selon les chiffres avancés par cette ONG. Pour les consommateurs, l'impact négatif de la contrefaçon se situe au niveau de la tromperie sur la qualité du produit. Ce camouflage peut s'avérer très dangereux pour la santé et la sécurité. Viala (2008) s'inscrit dans ce registre et analyse les nuisances du phénomène de la contrefaçon contre lequel tous les États s'attellent à renforcer leurs dispositifs pour se prémunir.

Ces différents travaux montrent, en effet, tout le caractère nuisible d'une pratique illicite de plus en plus en vogue et en expansion. Avec la mondialisation des échanges, les contrefacteurs profitent des failles du système juridique pour écouler illégalement leurs marchandises. C'est dans cette perspective que s'oriente notre recherche qui va porter sur l'évaluation de l'application des textes juridiques dans le cadre global de la lutte contre la contrefaçon. De nombreuses études ont été déjà menées et portent sur différentes orientations scientifiques dans ce domaine. La propriété intellectuelle constitue jusqu'à présent un des thèmes majeurs d'actualité sur le plan international en matière économique et juridique. Dans ce sens, Piriou(2001) met l'accent sur la légitimité des droits de propriété intellectuelle. Selon elle, l'attention portée par certains philosophes sur l'économie va, à partir du XVIII ${ }^{\text {ème }}$ siècle, tendre vers une reconnaissance du travail de l'auteur qui constitue, selon l'axiome de Locke, la source d'un droit naturel donnant naissance à un droit de propriété. Cette idée va influencer l'esprit des lois votées.

Castelain et Rebbot (2007) illustrent bien cette légitimité en mettant plus d'accent sur la loi adoptée le 29 octobre 2007 par l'Europe. La loi $\mathrm{N}^{\circ}$ 2007-1544 relative à la lutte contre la contrefaçon transpose, en effet, la directive 2004/48/CE du Parlement européen et du Conseil du 29 avril 2004 relative au respect des droits de propriété intellectuelle. Cette loi nouvelle vient harmoniser les dispositions préexistantes dans le droit concernant les personnes ayant qualité pour agir, les juridictions compétentes pour statuer sur les litiges relatifs aux atteintes portant sur les droits de propriété intellectuelle, et les régimes procéduraux mis à la disposition des victimes. Elle institue un véritable droit d'information au profit des requérants, ainsi qu'un arsenal procédural puissant, couplé à des sanctions dissuasives. Azéma (2014) estime que la loi du 11 mars 2014 renforçant la lutte contre la contrefaçon, s'inscrit dans une démarche tendant depuis plusieurs années à 
perfectionner l'arsenal juridique français tout en harmonisant les dispositions législatives d'un secteur à l'autre de la propriété intellectuelle. Cet auteur pense que cette loi améliore les dispositions relatives aux poursuites et aux sanctions de la contrefaçon et renforce les moyens d'action des douanes. Harms (2007) va au-delà de cette analyse et propose d'examiner la raison d'être et l'efficacité de l'application des droits de propriété intellectuelle au moyen de sanctions pénales.

Sur un volet relatif à la protection des logiciels, Djomga (2011) évoque le regroupement de certains pays d'Afrique francophone au sein de l'Organisation Africaine de la Propriété Intellectuelle (OAPI). Ceux-ci ont mis en place l'Accord de Bangui adopté le 2 mars 1977. Cet accord sert de référence pour chacun des États membres et a fait l'objet d'une révision le 24 février 1999, avec pour objectif de mettre la législation de l'OAPI en conformité avec les conventions internationales, notamment l'Accord sur les Aspects des Droits de Propriété Intellectuelle qui touchent au Commerce. Cet auteur relève comment les législations des pays membres de l'OAPI relatives au droit d'auteur sanctionnent les atteintes aux droits conférés aux développeurs et éditeurs de logiciels au titre de la contrefaçon sur le double plan civil et pénal.

En ce qui concerne la propriété industrielle, elle vise les brevets, les marques de produits, les dessins et modèles industriels, les modèles d'utilité, les marques de services, les noms commerciaux, les indications géographiques, ainsi que la répression de la concurrence déloyale. Sur cette question, la Convention de Paris, cet accord international, est la première grande mesure prise pour aider les créateurs à faire en sorte que leurs œuvres intellectuelles soient protégées dans d'autres pays. (Convention de Paris, 1883)

Wyssbrod (2008) aborde la question de la contrefaçon dans sa globalité. Pour lui, c'est un problème croissant, et enrayer cette activité illicite ne peut aboutir qu'en s'appuyant sur la législation. Les recherches de cet auteur vont ainsi porter sur le changement qu'a subi le droit des marques Suisse lors de la révision de la loi du $1^{\text {er }}$ juillet 2008. Soton (2013) met plus l'accent sur les conditions de la protection de la marque. La protection juridique de la marque porte sur des signes susceptibles de représentation graphique. Cela suppose que le signe choisi puisse être représenté visuellement au moyen de figures, de ligne ou de caractère, et ceci de façon claire, précise et complète.

Au regard de la recension des écrits on note que la protection des droits de propriété intellectuelle est un véritable sujet d'actualité. L'existence de nombreux textes de lois au niveau international exprime la volonté manifeste de chaque État dans la lutte contre la contrefaçon. Au plan national, plusieurs textes de lois ont été ratifiés et sont mis en application. En 
dépit de ces différents textes de lois, la contrefaçon continue de gagner du terrain. De nombreuses recherches ont été menées, cependant très peu se sont intéressées à l'évaluation des textes de lois en matière de lutte contre la contrefaçon.

Aujourd'hui, il convient de s'interroger sur l'impact réel de ces textes dans la lutte contre le phénomène ? Quel est l'état des lieux de l'application des textes juridiques en Côte d'Ivoire en matière de lutte contre la contrefaçon? Les acteurs ont-ils les moyens et jouent-ils pleinement leurs rôles ? C'est à ces questions que ce travail va tenter de répondre. Les objectifs assignés à cette étude sont les suivants :

- faire un état des lieux des différents textes de lois en matière de lutte contre la contrefaçon ;

- $\quad$ décrire le mode d'application de ces textes juridiques ;

- $\quad$ évaluer l'application de ces différents textes juridiques.

Dans un souci de précision, il nous a semblé adéquat de nous intéresser aux théories qui semblent les plus adaptées à la compréhension de l'objet. Ce sont la théorie du contrôle social de Cusson et la théorie économique du crime de Becker.

La théorie du contrôle social du crime (1983) de Cusson propose une typologie dans laquelle sont distingués les contrôles sociaux thérapeutiques, moraux, dissuasifs, ainsi que ceux qui sont fondés sur la justice et les rapports de réciprocité.

L'analyse de Becker $(1976,1977)$ est basée sur la rationalité des comportements et sur l'équilibre des marchés. Il élargit l'analyse économique à de nouveaux domaines en utilisant les outils de la théorie néoclassique (maximisation sous contrainte, équilibre du marché, stabilité des préférences, courbes d'indifférences).

\section{Methodologie}

\section{Site et participants à l'enquête}

L'étude se déroule à Abidjan, capitale économique de la Côte d'Ivoire. Cependant les recherches de terrain ont eu lieu dans les communes du plateau précisément à la Direction de la surveillance et des interventions des Douanes ivoiriennes et à Koumassi. Dans un souci de diversification des sources de données, l'enquête a porté sur 32 participants tirés sur la base d'un échantillonnage intentionnel et issus de tous les sites visités.

\section{Instruments de recueil de données}

Les données ont été recueillies par le biais de la recherche documentaire, de l'observation directe et du questionnaire. 


\section{Recherche documentaire}

Nous avons parcouru des centres de documentations de certaines institutions de la place. Dans cette perspective nous nous sommes rendus à la bibliothèque de l'UFR Criminologie de l'Université Felix Houphouët Boigny de Cocody et, aux centres de documentation de l'Assemblée Nationale et de l'Office Ivoirien de la Propriété Intellectuelle (OIPI). Sur ces différents sites, il s'agissait de recueillir de la littérature sur les textes de lois en lien avec la contrefaçon, les modalités d'application; de façon générale sur la lutte contre contrefaçon

\section{Observation directe}

Nous avons pu observer lors de notre passage à la Direction de la Surveillance et des Interventions (DSI) l'inaction des agents des Douanes vis-à-vis des différents rapports faisant état des trafics de la contrefaçon. En plus nous avons pu observer la résistance et la réticence des commerçants face à l'application des textes de lois.

\section{Questionnaire}

Nous nous sommes adressés aux trois (03) catégories d'échantillon de participants à notre étude qui sont :

les agents de la Douane et la Police Économique parce qu'ils représentent les principaux acteurs de la lutte contre la contrefaçon et de l'application des textes juridiques. En plus ce sont eux qui sont en mesure de nous expliquer la manifestation du phénomène de la contrefaçon. Dès lors, ils deviennent des interlocuteurs incontournables,

- Les commerçants pour connaître les motifs de l'existence de la contrefaçon et leur point de vue sur les textes de lois,

- La population civile pour savoir si elle a connaissance des textes de lois et connaître leur point de vue.

Les thématiques abordées tournaient autour de la connaissance de l'existence des textes de lois en matière de lutte contre la contrefaçon, l'application des textes de lois, la manifestation de la contrefaçon et les raisons de l'existence de la contrefaçon

\section{Analyse des données}

S'il est vrai que les données ont été recueillies avec un questionnaire, l'analyse de ces dites données sera faite d'un point de vue qualitatif. Ainsi nous avons analysé les discours, les mots et les différents expériences vécues par les enquêtés.

\section{Résultats}

L'exposé des résultats concerne la description des textes juridiques 
nationaux, la modalité d'application ainsi que l'évaluation de la mise en œuvre de ces textes.

\section{Description des textes juridiques nationaux}

En Côte d'Ivoire, la lutte contre la contrefaçon est un défi majeur à relever pour les autorités compétentes. C'est la raison pour laquelle la majorité des dispositions juridiques internationales concernant les droits de la propriété intellectuelle ont été ratifiées par l'État ivoirien.

\section{Présentation des textes juridiques}

Dix textes ont été analysés et se présentent comme suit dans le tableau ci-dessous :

Tableau : Différents textes de lois concernant la lutte contre la contrefaçon

\section{ORIENTATION \\ DES TEXTES \\ JURIDIQUES}

\section{TEXTES JURIDIQUES}

- décret $n^{\circ} 92-393$ du $1^{\text {er }}$ juillet 1992 relatif à l'indication de la provenance des tissus imprimés vendus en Côtes d'Ivoire.

Les textes relatifs à la protection des

droits de la

propriété

intellectuelle
- Circulaire $\mathrm{n}^{\circ} 1257 \mathrm{du} 26$ Janvier 2005 concernant les interdictions relatives aux importations et au dédouanement de marchandises aux bureaux frontières.

- loi n²013-865 du 23 Décembre 20132013 relative à la lutte contre la contrefaçon et le piratage, et à la protection des droits de propriété intellectuelle dans les opérations d'importation, d'exportation et de commercialisation de biens et services.

- loi n²016-555 du 26 juillet 2016 fixant les règles relatives à la protection du droit d'auteur et des droits voisins.

- décret $\mathrm{n}^{\circ}$ 2007-468 du 15 mai 2007 portant création, organisation, attributions et fonctionnement de la Direction de la Surveillance et des Interventions au sein de l'administration des Douanes

Textes relatifs à la création, organisation, attribution et au fonctionnement des différentes structures

- décret n²012-699 du 1er août 2012 portant création, attributions, organisation et fonctionnement du Comité National des Indications Géographiques et des Marques Collectives de la Côte d'Ivoire, en abrégé CMC. - décret n²014-420 du 09 Juillet 2014 fixe les attributions, l'organisation et le fonctionnement du Comité national de lutte contre la contrefaçon - décret $n^{\circ} 2015-271$ du 22 avril 2015 fixant les attributions, l'organisation et le fonctionnement du Bureau ivoirien du Droit d'Auteur.

- loi nº 1981-640 du 31 juillet 1981, instituant le Code pénal (modifiée par la Loi $\mathrm{n}^{\circ} 1995-522$ du 6 juillet 1995)

Textes relatifs aux sanctions -loi Nº64-291 du 1 août 1964 portant code des Douanes modifié par ordonnance $\mathrm{n}^{\circ} 88-225$ du 2 mars 1988

Le tableau ci-dessus montre les différents textes de lois concernant la lutte contre la contrefaçon. Nous avons identifiés dix (10) textes de lois dont 
4 textes relatifs à la protection des droits de la propriété intellectuelle, 4 textes relatifs à la création, organisation, attribution et au fonctionnement des différentes structures et 2 textes relatifs aux sanctions.

\section{Mesures contenues dans les textes}

\section{- Les textes relatifs à la protection des droits de la propriété intellectuelle}

Ces textes sont relatifs aux droits d'auteur et aux droits de la propriété industrielle. Ils permettent aux auteurs des droits de propriété intellectuelle d'avoir le monopole d'exploitation temporaire de ses biens et services sur tout l'ensemble du territoire.

- Textes relatifs à la création, organisation, attribution et au fonctionnement des différentes structures

Il s'agit des textes de lois organiques. Ces textes permettent de mettre en place les structures, leur attribution et leur mode de fonctionnement.

\section{Textes relatifs aux sanctions}

Ces textes instituent l'infraction et les modalités de répression. Ils permettent de caractériser la peine et les amendes qui doivent être infligées à celui qui transgresse la loi.

\section{Modalités d'application des textes juridiques}

Présentation des acteurs en charge d'appliquer les textes juridiques

Comité national de lutte contre la contrefaçon (CNLC)

Le CNLC est une Autorité administrative indépendante dotée de la personnalité morale et de l'autonomie financière. Ce comité a pour mission de lutter contre la contrefaçon sur toute l'étendue du territoire national. A ce titre, il est notamment chargé, en liaison avec les administrations concernées :

$\checkmark \quad$ d'entreprendre des actions préventives afin d'empêcher l'introduction sur le territoire national de marchandises pirates ou de contrefaçon ou susceptibles de porter atteinte, de quelque manière, à des droits de propriété intellectuelle et de mettre hors des circuits commerciaux, de tels produits ;

$\checkmark \quad$ de coordonner, de suivre et d'évaluer l'exécution des programmes publics nationaux, sous régionaux, régionaux et internationaux de lutte contre la contrefaçon;

$\checkmark \quad$ d'assister les pouvoirs publics, les administrations compétentes, les juridictions et les titulaires de droits de propriété intellectuelle dans la recherche, la constatation et la poursuite des infractions en ces matières en vue de leur répression;

$\checkmark \quad$ de participer à l'élaboration des programmes nationaux de lutte contre la contrefaçon et la piraterie ;

$\checkmark \quad$ de contribuer à la mise en place d'un cadre de coordination des 
actions des différents administrations et organismes publics ou privés actifs en matière de lutte contre la contrefaçon et la piraterie ;

$\checkmark \quad$ de participer à l'élaboration et au développement de la législation relative à la lutte contre la contrefaçon et la piraterie et d'émettre son avis sur les projets de textes législatifs et réglementaires qui peuvent lui être soumis en la matière ;

$\checkmark \quad$ de collecter les informations et de mettre en place un système statistique et d'information sur la contrefaçon et la piraterie ;

$\checkmark \quad$ d'élaborer des plans et programmes de communication et de sensibilisation sur la lutte contre la contrefaçon et le piratage ;

$\checkmark \quad$ de contribuer à la mise en œuvre des mécanismes susceptibles de développer la coopération internationale en matière de lutte contre la contrefaçon;

$\checkmark \quad$ de produire, chaque année, au plus tard le 30 mars, un rapport d'activités transmis au Ministre chargé de l'Industrie.

\section{Bureau ivoirien des droits d'auteur (Burida)}

Le Burida a vocation à gérer, à titre exclusif, tous droits dont l'exercice lui est confié en application de la loi n 96-564 du 25 juillet 1996 relative à la protection des œuvres de l'esprit et aux droits des auteurs, des artistes-interprètes et des producteurs de phonogrammes et vidéogrammes. Le Burida est notamment chargé:

$\checkmark \quad$ de délivrer les autorisations, en application du mandat reçu par l'adhésion des titulaires de droits ou en application d'accords bilatéraux ou de tout mécanisme de gestion collective obligatoire ou étendu, et de percevoir les rémunérations correspondantes;

$\checkmark \quad$ de percevoir et de répartir tous droits à rémunération reconnus par la loi ou par tout instrument juridique international, et notamment, la rémunération pour copie privée, la rémunération équitable, la rémunération pour reproduction par reprographie et le droit de suite ;

$\checkmark \quad$ d'assurer la gestion de tous les fonds constitués en application des textes législatifs et réglementaires en vigueur, à des fins culturelles et sociales au bénéfice de ses associés ;

$\checkmark \quad$ de promouvoir une action sociale en faveur des créateurs d'œuvres littéraires ou artistiques et des titulaires de droits voisins notamment par la création et la gestion d'un fonds social à leur profit ;

$\checkmark \quad$ de percevoir toutes sommes pouvant revenir à l'ensemble des professions qu'il représente, au titre d'une indemnisation conventionnelle ou judiciaire ;

$\checkmark \quad$ de gérer sur le territoire national les droits des ayants droit des organismes d'auteurs, d'artistes interprètes ou de producteurs étrangers dans le cadre des conventions ou accords conclus avec eux et d'exercer les droits 
des associés du BURIDA à l'étranger, dans le cadre de ces mêmes accords; $\checkmark \quad$ de promouvoir et de défendre les intérêts matériels et moraux de ses associés ou de leurs ayants droits ;

$\checkmark \quad$ d'ester en justice, de prendre toutes dispositions et d'accomplir tous actes destinés à la bonne réalisation de sa mission.

\section{Direction de la Surveillance et des Interventions}

Elle a pour fonction de lutter contre la contrefaçon en recherchant et saisissant toutes marchandises importées ou exportées faisant l'objet de contrefaçon.

\section{Application des textes juridiques}

\section{Saisine par le Bureau ivoirien des droits d'auteur}

Lorsqu'une action en contrefaçon a été engagée en justice directement par le titulaire des droits lui-même ou ses ayants droit, l'organisme de gestion collective dont ce titulaire de droit est membre, doit être mis en cause à l'instant. Les associations professionnelles d'ayants droit régulièrement constituées pour la défense des intérêts collectifs de leurs adhérents ont également qualité pour agir. En l'absence de personne justifiant d'un intérêt pour agir, notamment s'il n'y a pas d'ayants droit connus, en cas de vacance ou de déshérence, ou dans l'hypothèse où le titulaire du droit est hors d'état de manifester sa volonté, le ministre chargé de la Culture ou l'organisme de gestion collective habilité, peut saisir la juridiction compétente. Sans préjudice des droits de poursuite réservés aux officiers de police judiciaire, l'organisme de gestion collective est autorisé à désigner des représentants assermentés habilités à contrôler l'exécution des prescriptions de la présente loi sur le territoire national et à constater les infractions. Les autorités notamment de la Police nationale, des Douanes et de la Gendarmerie nationale sont tenues, à la demande des organismes de gestion collective, de prêter leur concours et, le cas échéant, leur protection à l'accomplissement de leurs missions. À la requête de tout titulaire de droits d'auteur ou de droits voisins, de ses ayants droit ou d'un organisme de gestion collective, les officiers de police judiciaire ou tout agent assermenté de l'organisme de gestion collective sont tenus de saisir les exemplaires constituant une reproduction illicite d'une œuvre, d'une prestation ou d'une fixation. A la requête des personnes mentionnées, le président du tribunal de première instance, ou le président de section peut ordonner, moyennant caution, s'il y a lieu la saisie en tous lieux, et même en dehors des heures prévues par le Code de procédure pénale, des exemplaires fabriqués, ou en cours de fabrication, d'une œuvre, d'une prestation ou d'une fixation illicitement reproduite; la suspension de toute fabrication, représentation ou exécution publique en cours ou annoncée constituant une contrefaçon ou un acte préparatoire à une 
contrefaçon. Le saisi ou le tiers saisi peut demander au magistrat qui l'a ordonnée de prononcer la mainlevée de la saisie ou d'en cantonner les effets, ou encore d'autoriser la reprise de la fabrication ou celle des représentations ou exécutions publiques sous l'autorité d'un administrateur constitué séquestre, pour le compte de qui il appartiendra, des produits de cette fabrication ou de cette exploitation. S'il est fait droit à la demande du saisi ou du tiers saisi, il peut être ordonné, à la charge du demandeur, la consignation d'une somme affectée à la garantie des dommages et intérêts auxquels l'auteur pourrait prétendre. Les titulaires de droits d'auteur ou de droits voisins et leurs ayants droit ainsi que les organismes de gestion collective pourront avec l'autorisation du président du tribunal de première instance, le juge de section ou le juge délégué compétent, obtenue sur requête, faire procéder par un ou plusieurs experts, que désignera ce magistrat, à la description des objets prétendus contrefaisants ou des faits de la contrefaçon et du matériel qui ont directement servi à les accomplir. Dans le cas d'un programme d'ordinateur ou d'une base de données contrefaisant, la saisiedescription peut se concrétiser par une copie.

\section{Saisine par le Comité national de lutte contre la contrefaçon}

La saisine des produits contrefaisants s'opère sur requête du détenteur d'un droit de propriété intellectuelle. Cette requête doit être adressée à l'administration des douanes qui suspend le dédouanement ou la mise en circulation des marchandises soupçonnées de porter atteintes à un droit de propriété intellectuelle tant à l'importation qu'à l'exportation. Le requérant transmet une copie de sa requête. Cette requête doit être accompagnée d'éléments de preuve établissant l'existence du droit; d'un exposé des motifs de la requête et en particulier des éléments de preuve de l'atteinte à un droit de propriété intellectuelle; d'une description détaillée des marchandises couvertes par le droit de propriété intellectuelle avec le cas échéant un échantillon du produit authentique ; de tous renseignements concernant le requérant et son lieu de résidence et d'une procuration si le détenteur est représenté par un mandataire. L'administration des Douanes dispose d'un délai de trois jours à compter de la date de réception de la requête pour y donner suite. Lorsqu'il est établi que les marchandises sont de contrefaçon, ou portent autrement atteinte à un droit de propriété intellectuelle, l'Administration des Douanes suspend le dédouanement ou la mise en circulation des marchandises. Ensuite elle fournit au détenteur du droit, à sa demande, les noms et adresses, selon les cas, de l'exportateur, de l'importateur, du propriétaire ou du destinataire, ainsi que les renseignements relatifs à la quantité des marchandises mises en cause. Le requérant ou la victime de la contrefaçon a une option entre la saisine de la juridiction pénale ou de la juridiction civile. 
- Saisine par la Direction de la Surveillance et des Interventions

L'importation, l'exportation, mais aussi la circulation ou la détention en tout point du territoire de marchandises présentées notamment sous une marque contrefaite ou un dessin ou un modèle contrefait, constituent une prohibition douanière absolue. Par conséquent le service des douanes peut procéder à la saisie des produits contrefaits à l'occasion d'un contrôle.

\section{Sanctions encourues Au niveau pénal}

Conformément à la loi n²016-555 du 26 Juillet 2016 relative au droit d'auteur et aux droits voisins, toute atteinte à l'un quelconque des droits moraux et patrimoniaux constitue le délit de contrefaçon. Est puni d'un emprisonnement d'un an à dix ans et d'une amende de 500000 à

5000000 de francs, ou de l'une de ces peines seulement

La tentative du délit de contrefaçon est punissable. Les peines sont portées au double lorsque l"auteur de l'infraction est le co-contractant du titulaire du droit violé.

Le code Pénal ne prévoit qu'une peine d'amende de 100000 à 1000 000 de francs s'il est établi que l'atteinte à la propriété artistique et littéraire est une contrefaçon. La peine est d'un mois à un an d'emprisonnement et de 200000 à 2000000 de francs d'amende s'il est établi que le coupable s'est livré habituellement aux actes visés au point présent.

Sont punis d'une amende de 100000 à 1000000 de francs et d'un emprisonnement de trois mois à trois ans ou de l'une de ces deux peines seulement s'il est établi qu'il y a eu contrefaçon et fraude en matière commerciale.

\section{Au niveau civil}

Les personnes dont le droit reconnu, a été violé, ont le droit d'obtenir le paiement, par l'auteur de la violation, de dommages-intérêts en réparation du préjudice subi par elles en conséquence de l'acte de violation, ainsi que le paiement des frais occasionnés par l'acte de violation, y compris les frais de justice. Pour fixer les dommages et intérêts, la juridiction prend en considération distinctement : les conséquences économiques négatives de l'atteinte aux droits, dont le manque à gagner et la perte subie par la partie lésée; le préjudice moral causé à cette dernière et les bénéfices réalisés par l'auteur de l'atteinte aux droits, y compris les économies d'investissements intellectuels, matériels et promotionnels que celui-ci a retirées de l'atteinte aux droits. Toutefois, la juridiction peut, à titre d'alternative et sur demande de la partie lésée, allouer à titre de dommages et intérêts une somme forfaitaire. Cette somme est supérieure au montant des redevances ou droits qui auraient été dus si l'auteur de l'atteinte avait demandé l'autorisation 
d'utiliser le droit auquel il a porté atteinte. Cette somme n'est pas exclusive de l'indemnisation du préjudice moral causé à la partie lésée.

\section{Évaluation de l'application des textes juridiques}

Il s'agit, ici, après étude, de dégager les forces et les faiblesses dans la mise en œuvre des textes juridiques en vigueur dans la lutte contre le phénomène de la contrefaçon en Côte d'Ivoire.

\section{Forces}

La seule existence déjà des textes juridiques contre le phénomène de la contrefaçon est un mérite. Ce dispositif constitue à priori un moyen de dissuasion sur le territoire ivoirien pour les auteurs de contrefaçon.

\section{Auteurs des textes juridiques}

Les lois, les décrets d'application et autres arrêtés sont l'œuvre de la présidence de la République et du Gouvernement, mais aussi de l'Assemblée nationale. A ces institutions de la République s'ajoutent de hauts responsables de l'Administration publique (douanes) qui interviennent dans le processus de mis en œuvre du dispositif juridique créé à travers les circulaires, les notes de service, etc. Que les pouvoirs publics, au plus haut sommet, c'est-à-dire, le Président de la République et son gouvernement ainsi que l'Assemblée nationale, soient à l'initiative des lois contre la contrefaçon, cela est un indicateur indéniable qui montre l'implication des autorités dans la lutte contre le phénomène.

\section{Textes juridiques}

La loi 2013-865 du 23 décembre 2013 est un dispositif qui engage formellement la lutte contre le phénomène de la contrefaçon. La prise du décret $\mathrm{N}^{\circ} 2014-420$ du 09 Juillet 2014 portant attributions, organisation et fonctionnement du Comité national de lutte contre la contrefaçon, matérialise la mise en œuvre de ladite loi, qui crée ce Comité. Les arrêtés ministériels, notes de services et circulaires se référant à ce décret, et qui déploient sur le terrain les hommes et les moyens, traduisent les actions qui résultent de ce dispositif juridique. Nous avons aussi la loi n²016-555 du 26 juillet 2016 qui fixe les règles relatives à la protection du droit d'auteur et des droits voisins ainsi que le décret $n^{\circ} 2015-271$ du 22 avril 2015 fixant les attributions, l'organisation et le fonctionnement du Bureau ivoirien du Droit d'Auteur.

\section{Faiblesses}

\section{Difficultés d'application des textes}

La lutte contre la contrefaçon se heurte à de très sérieux problèmes 
juridiques et d'exécution des décisions. Montrant les difficultés auxquelles ils sont confrontés le Directeur Général du commerce intérieur K, A. affirme: " Il y a des fraudeurs qui sont installés de manière solide dans certaines régions où ils contribuent de façon efficace à la vie économique. Ils sont des barons dans ces régions et parfois intouchables. Quand vous y allez, vous êtes quelques fois menacés. Quand vous faites des saisies, vous êtes parfois confrontés à des interventions pour empêcher les poursuites; ils sont relativement protégés 》. Lorsque les procédures ne sont pas rendues inopérantes par la volonté de préserver les intérêts locaux, l'impossibilité légale pour les agents assermentés de pénétrer dans des locaux sans le consentement des intéressés. Selon le Colonel des Douanes B, L. : «la loi permet à la Cellule de prévention et d'intervention du Comité national de lutte contre la Contrefaçon de s'autosaisir. Mais, même s'il s'autosaisit, il $n^{\prime}$ avait pas le droit d'ester en justice. Ce droit n'appartenant uniquement qu' au titulaire du droit dont la marque a été frauduleusement atteinte». Cela montre les limites des pouvoirs des agents assermentés et l'existence d'une certaine barrière entre eux et les titulaires de droits. Nos recherches ont montré qu'il existe un réel manque de collaboration entre ces deux entités.

\section{Caducité de certains textes juridiques}

Le code des Douanes ivoiriennes n'est pas adapté aux exigences du commerce international. En effet, aucun article ne fait mention de façon plus explicite des infractions liées à la contrefaçon qui est devenue aujourd'hui un fléau avec de graves conséquences. Les saisines effectuées sont le plus souvent sur la base des articles 32 et 33, qui sont relatifs aux prohibitions des marques. C'est le cas du décret $n^{\circ} 92-393$ du $1^{\text {er }}$ juillet 1992 relatif à l'indication de la provenance des tissus imprimés vendus en Côtes d'Ivoire. Ce décret stipule que la vente des tissus imprimés dont la provenance n'est pas indiquée sur lesdits produits, est prohibée en Côte d'Ivoire. Cela veut dire que l'indication de la provenance doit être lisiblement inscrite sur la lisière des imprimés et comporter le nom du pays d'origine, l'identité de l'entreprise fabricante et le numéro du dessin imprimé. Ce décret est caduc dans la mesure où on ne parle plus de prohibitions. Mais, on parle maintenant en thème de contrefaçon. Le code pénal est dépassé et les peines encourues et les amendes ne sont pas dissuasives.

\section{Failles de certains textes}

La loi n²013-865 du 23 Décembre 2013 créant le Comité national de lutte contre la contrefaçon (CNLC) présente des insuffisances dans la mesure où elle stipule, à son article 13, ceci : "Sans préjudice de la protection des renseignements confidentiels, l'administration des douanes peut autoriser le détenteur d'un droit de propriété intellectuelle, l'importateur ou 
l'exportateur à examiner les marchandises dont le dédouanement ou la mise en circulation est suspendu. L'administration des douanes peut également autoriser le prélèvement des échantillons en vue de vérifier, par tout moyen, si les marchandises sont de contrefaçon ou portent autrement atteintes à un droit de propriété intellectuelle ».

Article 19: "La responsabilité de l'administration des douanes est engagée lorsque la suspension d'office du dédouanement d'une marchandise portant atteinte à un droit de propriété intellectuelle est injustifiée, sauf si elle apporte la preuve qu'elle a agi de bonne foi »

Les textes de loi doivent permettre aux responsables de l'administration des douanes d'avoir un délai bien précis pour pouvoir réquisitionner la marchandise. Ce délai doit s'étendre sur toute la durée du processus pour permettre aux agents assermentés de travailler en toute quiétude.

En ce qui concerne le décret $n^{\circ} 92-393$ du $1^{\text {er }}$ juillet 1992 relatif à l'indication de la provenance des tissus imprimés vendus en Côtes d'Ivoire, texte qui concerne la vente des tissus imprimés dont la provenance n'est pas indiquée sur lesdits produits, cette loi est censée protéger les fabricants de pagne contre la contrefaçon. Mais, elle ne mentionne rien sur le phénomène. Ce décret n'a pas l'effet voulu dans son application.

\section{Non-implication des personnes concernées par les textes juridiques}

Les textes juridiques ne peuvent avoir qu'un effet de communication quand les auteurs de ces dispositifs ne traduisent pas véritablement leur volonté de mener le combat de la lutte contre la contrefaçon. Il ne suffit pas seulement de voter des lois ou de prendre des mesures, mais encore faut-il que les initiateurs de ces dispositifs les accompagnent d'une politique de divulgation pour les rendre plus dissuasifs et impliquer le maximum de personnes dans leur mise en œuvre. Soutenu par le Directeur Général du commerce intérieur A.K. affirme: "qu'il faut sensibiliser l'ensemble des acteurs aux textes, ce qui permettra aux industriels et aux commerçants d'être bien imprégnés et de connaître les mécanismes pour se protéger ».

\section{Moyen d'action en matière d'application des lois}

Les structures assermentées à compétence nationale ne peuvent pas appliquer correctement les textes juridiques sur l'ensemble du territoire parce qu'elles sont basées uniquement Abidjan. Le Colonel des Douanes B, L. affirme : "qu'avec une petite cellule de 7 à 8 personnes, on ne peut pas lutter contre la contrefaçon sur l'ensemble du territoire national ». Compte tenu des tâches à exécuter, cet effectif s'avère insignifiant pour couvrir l'ensemble du territoire national.

Sur le plan logistique, selon le Directeur Général du commerce 
intérieur K, A. : "Ils ont besoin de renforcer les moyens matériels ainsi que le capital humain pour être plus efficace ». Il existe un véritable abîme entre l'étendue de la contrefaçon et les moyens dont disposent les agents en charge d'appliquer les textes de lois. L'application des textes juridiques contre la contrefaçon nécessite de gros moyens financiers et matériels. Les agents en charge en ont suffisamment besoin pour faire face à des impératifs en terme de déploiements de personnels, de dotations en matériels roulants, de sécurité et d'équipements de pointe, voire de recyclages permanents pour adapter la lutte aux moyens modernes de détection, etc. Pour le Colonel des Douanes B, L. : "le budget n'est pas suffisant pour permettre à ces agents d'être opérationnels sur le terrain ».Ces difficultés vont occasionner, parfois, la corruption de certains agents assermentés dans la lutte contre la contrefaçon. Tout ceci montre un manque de suivi dans l'application des textes votés par les organes désignés plus haut.

\section{Discussion et conclusion}

La présente étude a porté sur l'évaluation de l'application des textes juridiques en vigueur en matière de lutte contre la contrefaçon en Côte d'Ivoire. L'objectif est d'évaluer la mise e œuvre des différents textes de lois en vigueur. L'étude a révélé l'existence de (10) dix textes de lois mis en application par le Comité national de lutte contre la contrefaçon, le Bureau ivoirien des droits d'auteur et la Direction de la surveillance et des interventions des Douanes ivoiriennes. La problématique au fondement de cette étude était centrée sur l'effectivité de l'application de ces différents textes de lois. Les résultats montrent que lesdits textes de lois rencontrent des difficultés dans leur l'application sur le terrain. Ces difficultés sont liées à la qualité même de la norme qu'il convient d'appliquer, mais aussi, aux retards générés par la lourdeur dans l'application des textes. Nous pouvons aussi faire allusion à l'absence ou l'insuffisance de volonté politique. La caducité de certaines lois favorise l'action des criminels qui agissent en toute impunité. De même, des failles dans certaines lois et la faiblesse des sanctions motivent les contrefacteurs à exercer voire étendre sereinement leurs activités criminelles. La non-implication des personnes concernées dans la conception des lois votées entrave également la lutte contre la contrefaçon. En effet, les opérateurs économiques devraient être consultés pour donner leur avis dans la conception et sur la mise en œuvre des textes juridiques existants. En plus, avec l'évolution de la contrefaçon, les moyens dont disposent les acteurs en charge d'appliquer les lois sont insuffisants. Les ressources financières, les moyens techniques, l'expertise et la formation du personnel font cruellement défaut. Ces résultats valident la théorie du contrôle social de Cusson (1983). Les criminels font de la contrefaçon leur activité parce qu'elle procure du profit et du plaisir si le contrôle social est 
défaillant à travers les difficultés dans l'application des textes juridiques. L'étude prend aussi en compte la théorie économique du crime conçue par Becker, G. (1976,1977). Selon cette théorie, les individus se lancent dans une activité criminelle à cause des coûts et des avantages et parce que les peines ne sont pas appliquées. Cette étude présente cependant des limites. Nous avons pris en compte que deux (2) communes dans le District d'Abidjan et le nombre d'enquêtés est réduit. Ces résultats sont en partie basés sur la documentation et les propos des enquêtés. Il serait plus intéressant que dans les prochains travaux, les recherches s'effectuent sur tout l'ensemble du District d'Abidjan. À partir de ces résultats, nous proposons que les peines soient plus dissuasives et que les amendes très élevées. Les moyens doivent être mis à la disposition des agents assermentés pour appliquer rigoureusement les lois. Ceux-ci devront disposer de pouvoir plus coercitifs, voire répressifs pour traquer contrefacteurs et marchandises contrefaites. Par ailleurs, il doit y avoir une franche collaboration entre les opérateurs économiques et les agents en charge d'appliquer les lois.

\section{References:}

1. Becker, G. (1976,1977). Approche économique du crime. Paris : Dalloz

2. Cusson, M. (1983). Le contrôle social du crime. Paris: Presses Universitaires de France. Collections Sociologies.

3. Azéma, J. (2014). Agrégé des Facultés de Droit Directeur honoraire du Centre Paul Roubier; Revue Lamy de la concurrence n ${ }^{\circ} 93$, pp. 18-23.

4. Djomga, C. (2011). La contrefaçon des logiciels dans l'espace OAPI. Étude comparée de l'Accord de Bangui Révisé et des Législations du Sénégal, du Gabon, de la Côte d'Ivoire et du Cameroun. Yaoundé, Ed : ISIS.

5. B, Ladji. (2013). Lutte anti-contrebande et anti-contrefaçon dans le secteur textile en Côte d'ivoire (cas du pagne wax sur les marches abidjanais). Revue Africaine de Criminologie ${ }^{\circ} 12$. Abidjan: Éditions Universitaires de Côte d'Ivoire, pp. 8-19.

6. Bhumindr, B. (2012). La Contrefaçon des droits de propriété intellectuelle : Étude comparative en droits français et thaïlandais. Thèse de doctorat en Droit à l'Université Panthéon-Assas, France.

7. Castelain, J \& Rebbot, N. (2007). La loi de lutte contre la contrefaçon: première lecture. Paris: LEGIPRESSE revue mensuelle du droit de communication n ${ }^{\circ} 247$, pp. 167-171.

8. Wyssbrod, V. (2008). L'usage prive de contrefaçons et les sanctions pénales en droit des marques. Mémoire/Thèse de Master en droit Université de Neuchâtel, Suisse. 
9. Kazon, D. (2015). Étude évaluative de la protection des enfants en conflits avec la loi en Côte d'ivoire. Journal Africain de Communication Scientifique et Technologique, $\mathrm{N}^{\circ} 28,3645-3652$.

10. Harms, L. (2007). L'application des droits de propriété intellectuelle au moyen de sanctions pénales: une évaluation. Genève, Rapport présenté par le Comité consultatif sur l'application des droits.

11. Audace Institut Afrique (2013). Séminaire sur l'impact du non-respect de la propriété intellectuelle sur la santé publique et l'économie. Abidjan: AIA.

12. Krissi, C. (2010). Contrefaçon des médicaments et stratégies technologiques pour sécuriser la chaîne d'approvisionnement pharmaceutique. Mémoire de maîtrise, École polytechnique de Montréal.

13. Ponticelli, L. (2016). Contrefaçon dans l'édition littéraire aux XVIe et XVIIe siècles : étude de la pratique autour d'un corpus de livres anciens de la Bibliothèque municipale de Lyon. Mémoire de master 2 professionnel, Université de Lyon.

14. Hyeans, A. (2011). La contrefaçon dans le monde: entre dangers, profits et perspectives. Cahiers de la sécurité $n^{\circ} 15$. Éditions: La documentation française.

15. Mourad, S. (2014). Les effets de la contrefaçon sur le comportement d'achat de la marque de luxe en termes d'expérience et de relation à la marque. Thèse en Sciences de Gestion, Université de Grenoble.

16. Maitre, P. \& Perrino, M. (2007). Contrefaçon et Ostentation. Paris : Revue d'économie industrielle $\mathrm{n}^{\circ} 117$.

17. Viala, C. (2008). Livre noir de la contrefaçon. Paris: Hugo et Compagnie.

18. Namoya, S, Aka, Claver, Péhé, Émeline \& Somian, Isabelle. (2017). Lutte contre la fraude et la contrefaçon. Abidjan: Fraternité Matin $\mathrm{n}^{\circ} 15867$, pp. 2-3. 